\title{
评 述地球内部水专题
}

\section{水与地幔的部分熔融}

\author{
倪怀玮*, 张力, 郭璇
}

中国科学院壳幔物质与环境重点实验室, 中国科学技术大学地球和空间科学学院, 合肥 230026

*E-mail: hwni@ustc.edu.cn

收稿日期: 2015-07-20; 接受日期: 2015-12-03; 网络版发表日期: 2016-02-18

国家自然科学基金项目(批准号: 41590622, 41473058)、高等学校学科创新引智计划项目、中央高校基本科研业务费专项项目和中组部“青 年千人计划”项目资助

摘要＼cjkstart水对于地幔的部分熔融发挥了关键作用。地幔岩浆作用主要发生在板块边界(俯冲带和洋中脊)和若干 板内热异常区域。在大洋俯冲带，俯冲板片释出的水可以诱发上覆地幔楔甚至板片自身发生熔融，导致弧岩浆 作用，也有可能形成超临界流体。板片熔融和产生超临界流体的物理化学条件仍存在争议.在洋中脊和板内热 异常区域, 水和 $\mathrm{CO}_{2}$ 使上涌地幔发生熔融的起始深度增加, 熔融比例增大. 在地球深部层圈边界可能发生低程度 的地幔熔融，如岩石圈和软流圈边界、上地幔和过渡带边界、过渡带和下地幔边界等，其成因一般认为与边界两 侧矿物储水能力的差异有关. 水可以促进地幔岩石熔融的根本原因在于水是一种不相容组分, 强烈倾向于富集 在硅酸盐熔体相(矿物-熔体的水分配系数远小于 1), 从而令其吉布斯自由能降低. 前人对水在橄榄石、辉石和石 榴石等地幔矿物与熔体之间的分配行为已经研究得比较充分, 但水对硅酸盐熔体密度和迁移性质的影响还需要 更进一步的高温高压实验和计算制约.

关键词水，地幔，部分熔融，硅酸盐熔体，分配系数，超临界流体

\section{1 引言}

地幔岩石中通常含有一定数量的水，其丰度以 $\mathrm{H}_{2} \mathrm{O}$ 质量分数形式表示一般仅为 $50 \sim 1000 \mathrm{ppm}$ (Bell和 Rossman, 1992; Michael, 1995; Dixon等, 2002; Workman 和Hart, 2005). 除了以羟基 $(\mathrm{OH})$ 形式赋存在角闪石、云 母和高密度含水镁硅酸盐等含水矿物中, 少量的H还 可以在长石、橄榄石(及其高压变体瓦兹利石和林伍 德石)、辉石、石榴石、布里奇曼石(Bridgemanite，即 镁钙钛矿)和铁方镁石等名义上无水矿物中以点缺陷
的方式存在，主要表现为 $\mathrm{OH}$ 种型，在长石中也可能 表现为 $\mathrm{H}_{2} \mathrm{O}$ 种型(Beran和Libowitzky，2006; Johnson, 2006; Skogby, 2006; Smyth, 2006). 虽然只是地幔中 的一种微量或次要组分，水却在地幔的几乎所有熔 融过程中都扮演了至关重要的角色，这与水在矿物 和硅酸盐熔体之间的分配行为之间存在密切的关系. 在总结高温高压岩石学实验最新研究进展的基础上, 结合天然岩石和矿物样品水含量分析结果, 本文将 对水在地幔熔融过程中所起的作用以及水在矿物和 熔体之间的分配系数展开系统的讨论，简要分析水

中文引用格式: 倪怀玮, 张力, 郭璇. 2016. 水与地幔的部分熔融. 中国科学: 地球科学, 46: 329-340, doi: 10.1360/N072015-00217

英文引用格式： Ni H W, Zhang L, Guo X. 2016. Water and partial melting of Earth's mantle. Science China Earth Sciences, 59: 720-730, doi: 10.1007/s11430-015-5254-8 
对硅酸盐熔体的密度等物理性质的影响, 并对相关 领域的研究前景作出展望.

\section{2 水与幔源岩浆作用}

地幔熔融导致的岩浆作用大多发生在板块边界, 主要包括汇聚型的俯冲带和离散型的洋中脊; 另有 少量的板内岩浆作用发生在温度异常高的区域, 形 成洋岛玄武岩和大陆溢流玄武岩. 虽然不同构造环 境的含水性存在很大差异, 但水对于地幔熔融过程 都施加了关键影响.

\section{1 大洋俯冲带}

前人对俯冲带流体活动进行了广泛深入的研究, 认为大陆俯冲带相对缺乏流体活动(Rumble等, 2003; Zheng等, 2003). 与此相反, 大洋俯冲带是一个典型 的富水环境, 洋底沉积物与经过热液蚀变的洋壳和 岩石圈地幔中都含有大量的水. 随着俯冲过程中温 度和压强的升高, 俯冲板片中含水矿物发生分解, 释 放出富水流体(Poli和Schmidt, 2002). 低密度的流体 向上运移, 与上覆地幔楔橄榄岩反应形成富化富集 的交代岩, 由此部分熔融就产生玄武质或碧玄质弧
岩浆, 这是目前广泛接受的大洋俯冲带之上岩浆形 成机制(Stern, 2002). 虽然不同研究者的实验或模型 结果之间存在一定差别(Katz等, 2003; Grove等, 2006; Green等, 2010, 2014; Till等, 2012), 但它们均表明在 $50 \sim 150 \mathrm{~km}$ 深度, 水饱和条件下地幔橄榄岩的熔融温 度要比无水条件下低 300 600K, 而且在压强 $<1.5$ $\mathrm{GPa}$ 时熔融温度随深度的增加而降低(图1).

由于富水岩石体系容易在较低的温度下发生熔 融(Hermann等, 2006; Zheng等, 2011), 俯冲板片表层 的玄武质洋壳自身是否发生熔融也受到广泛关注 (Prouteau等, 2001; Hack等, 2007; Kimura和Nakajima, 2014). Kessel等(2005a)通过淬火方法确定了玄武岩水体系的固相线, 并发现固相线终止于 $1050{ }^{\circ} \mathrm{C}$ 和 5.3 $\mathrm{GPa}$, 即第二临界端点(Second critical endpoint)所处 的位置(图2). Mibe等(2011)利用原位同步辐射X射线 成像技术得到的玄武质熔体-流体临界曲线与固相线 相交给出的第二临界端点位于 $770^{\circ} \mathrm{C}$ 和 $3.4 \mathrm{GPa}$, 明显 低于Kessel等(2005a)的结果. 造成这种不一致性的原 因可能在于, Mibe等(2011)误认为体系中只存在一种 液相就一定表明它处于超临界条件, 从而低估了临 界曲线对应的压强.

确定玄武岩-水体系的第二临界端点对应的温度

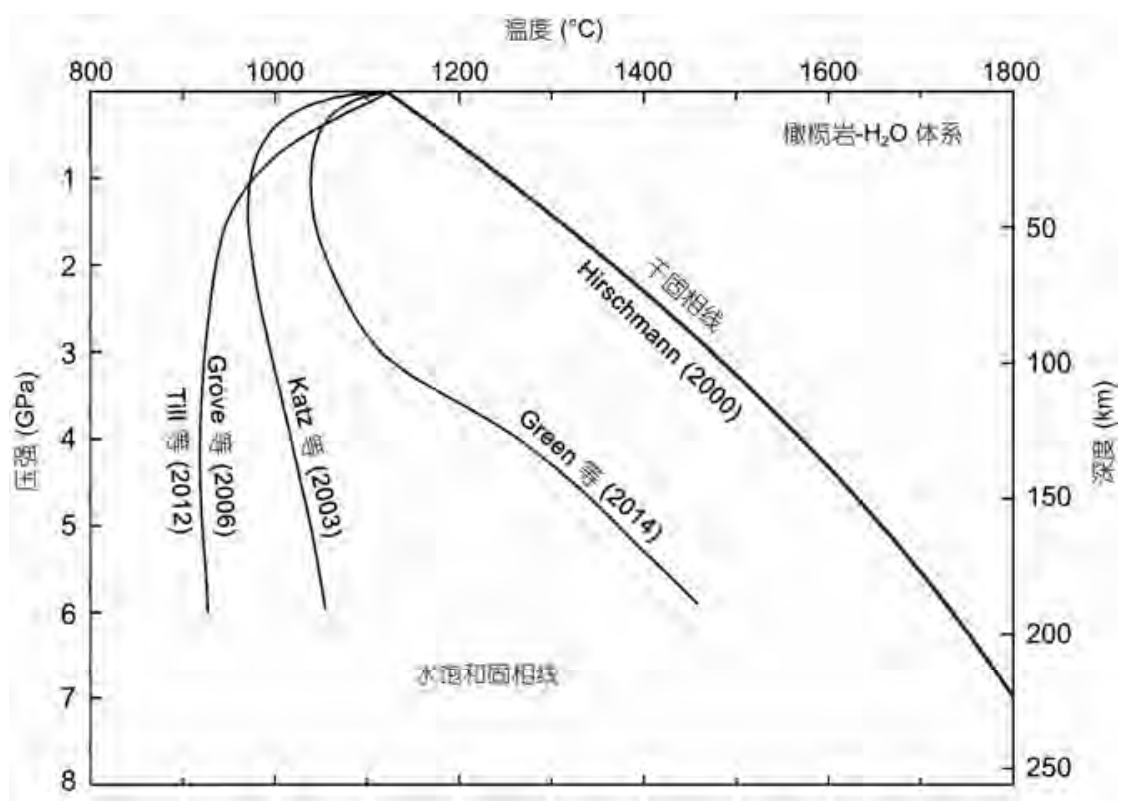

图 1 无水和水饱和条件下的橄榄岩- $\mathrm{H}_{2} \mathrm{O}$ 体系相图

综合无水(Hirschmann, 2000)和水饱和条件(Katz 等, 2003; Grove 等, 2006; Till 等, 2012; Green 等, 2014)研究结果绘制, 未考虑存在含水矿物的 情形 


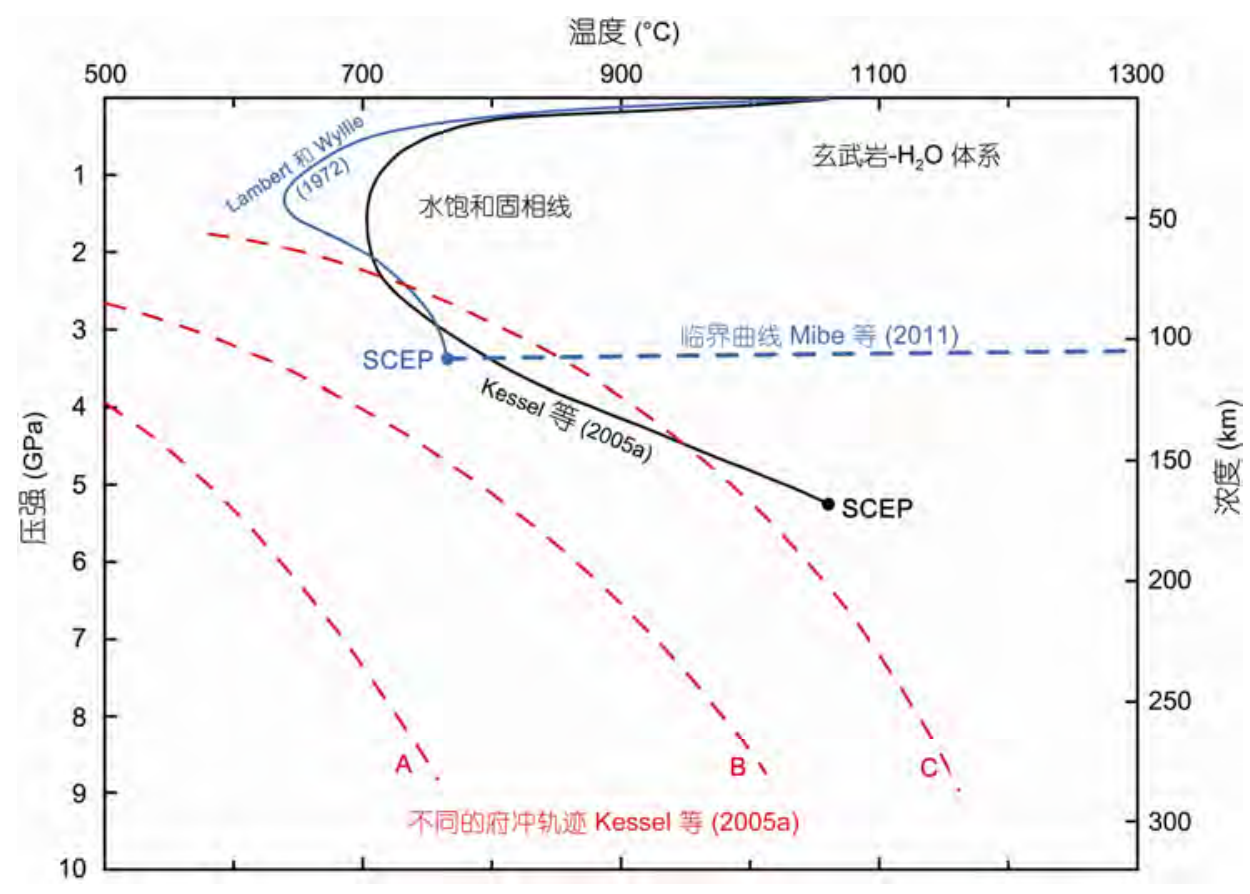

图 2 水饱和条件下玄武岩 $-\mathrm{H}_{2} \mathrm{O}$ 体系的固相线和第二临界端点

实线代表水饱和固相线, 蓝色虚线代表熔体-流体临界曲线, SCEP 代表第二临界端点, 3 条红色虚线代表不同的俯冲轨迹, 根据 Kessel 等 (2005a)和 Mibe 等(2011)修改

$T_{\mathrm{c}}$ 和压强 $P_{\mathrm{c}}$ 十分重要, 因为这与超临界流体形成的物 理化学条件密切相关. 一般将温压条件位于第二临 界端点之上的所有熔/流体都统称为超临界流体. 在 比 $P_{\mathrm{c}}$ 低的压强下, 熔体和流体是成分和物理化学性 质存在明显差异的两相, 富水的岩石体系在升温 并达到固相线时会发生一次由富水流体转化为含水 熔体的熔融事件 (图3a). 在比 $P_{\mathrm{c}}$ 高的压强下, 熔体与 流体之间的混溶隙完全消失, 在升温时富水流体中 的硅酸盐物质逐渐增多, 在温度到达 $T_{\mathrm{c}}$ 后演化为同时 富含水和硅酸盐的中间成分超临界流体(图3b); 继续 升温导致流体中的水含量进一步降低, 演化为含水 超临界流体 (单从成分上看也可视为含水熔体). 超临 界流体(尤其是中间成分的超临界流体) 是一种成 分介于富水流体和硅酸盐熔体之间的特殊流体, 其 物理化学性质与富水流体或硅酸盐熔体都存在显著 差别.

对于沿着较冷 $P-T$ 轨迹(如图 2 中的 $\mathrm{A}$ 和 $\mathrm{B}$ )俯冲的 古老洋壳来说, 其 $P-T$ 轨迹与固相线不相交, 在不足 100 160km深度(对应于3.4 5.3GPa) 释放的是富水流 体, 其所含硅酸盐溶质一般不超过 $20 \mathrm{wt} . \%$; 即便俯冲
至 $P>P_{\mathrm{c}}$ 所对应的深度范围, 能否形成超临界流体还 取决于温度是否达到或超过 $T_{\mathrm{c}}$. 只有沿着较热 $P-T$ 轨 迹(如图2中的C)俯冲的年轻洋壳, 其 $P-T$ 轨迹才会与 固相线相交于约 $700^{\circ} \mathrm{C}$ 和 $2 \mathrm{GPa}$, 即在约 $60 \mathrm{~km}$ 深度发 生部分熔融, 这样产生的往往是具有显著元素地球 化学特征的埃达克质岩浆 (Defant 和 Drummond, 1990). 按照Kessel等(2005a) 的实验结果, 热的洋壳 可能在约 $130 \mathrm{~km}$ 深度再次跨越固相线, 导致熔体转化 为一种过碱性的流体，继而在更高的温压条件下转 化为超临界流体.

由于超临界流体兼具流体黏度低、易流动与熔体 润湿性强的特点(Audétat和Keppler, 2004), 很多学者 设想超临界流体是俯冲带元素(特别是Zr等高场强元 素)迁移和地幔交代作用的重要介质(Shen和Keppler, 1997; Kessel等, 2005b; Adam等, 2014; Zheng和 Hermann, 2014). 然而Manning(2004)认为, 超临界流 体只在十分有限的温压区间才会形成，而且在脱离 俯冲板片后无法运移太长距离, 会发生相分离形成 含水熔体和富水流体. 关于玄武岩-水体系的相关系、 板片熔体和超临界流体的形成条件及其对元素迁移 

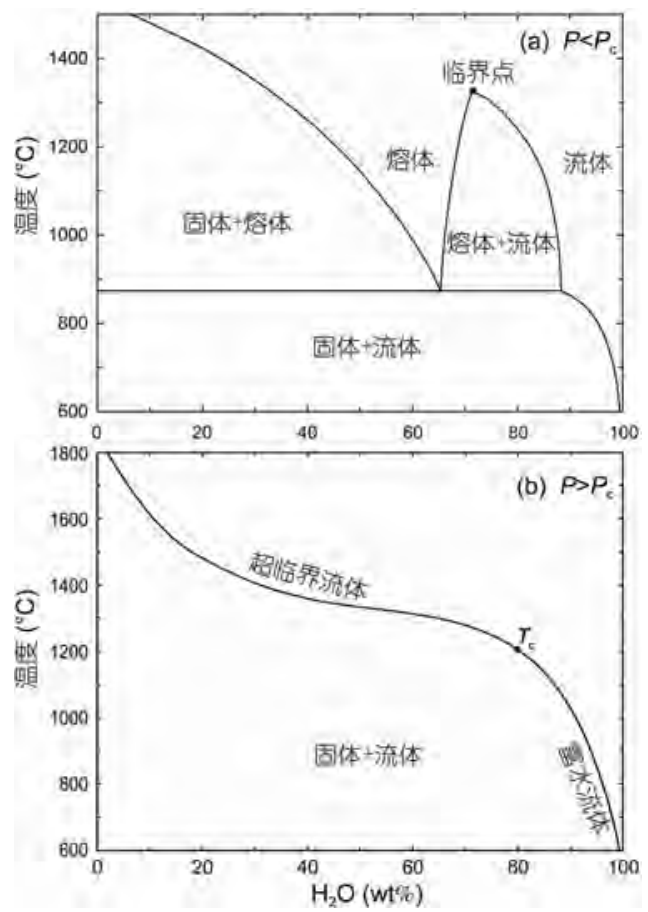

图 3 玄武岩- $\mathrm{H}_{2} \mathrm{O}$ 体系等压相图

横坐标为体系中水的质量百分数; (a) 对应压强为 $4 \mathrm{GPa}$, 低于第二 临界端点 $P_{\mathrm{c}}=5.3 \mathrm{GPa}$; (b) 对应压强为 $6 \mathrm{GPa}$, 位于第二临界端点压强 之上, $T_{\mathrm{c}}$ 为第二临界端点温度. 根据 Kessel 等(2005a)修改
所起作用等方面尚有很多问题函待澄清.

\section{2 洋中脊和板内热异常区域}

洋中脊是一个相对贫水的地质环境，不存在富 水的流体相. 洋中脊玄武岩(MORB)的形成机制主要 为地幔减压熔融, 即地幔沿绝热地温梯度上涌, 在约 $65 \mathrm{~km}$ 深度穿越固相线(图4). 然而微量元素地球化学 方面的证据表明，熔融早在超过 $100 \mathrm{~km}$ 深度的石榴石 稳定域就已经发生了(Salters和Hart, 1989)。起始熔融 深度的增加与水的作用密切相关(Aubaud等, 2004; Hirschmann等, 2009). 图4显示, 当地幔水含量为 50 ppm时，起始熔融深度增至 $80 \mathrm{~km}$; 地幔水含量为 200 ppm时，起始熔融深度增至 $115 \mathrm{~km}$. 水不仅令熔融提 前发生，也导致地幔部分熔融程度增大(Hirschmann 等, 2009).

上地幔中还含有几十至几百 $\mathrm{ppm}$ 的 $\mathrm{CO}_{2}($ Salters和 Stracke, 2004; Workman和 Hart, 2005; Dasgupta, 2013). $\mathrm{CO}_{2}$ 对地幔熔融的作用与水类似(图4), 它可以 使橄榄岩的起始熔融深度增至 $180 \mathrm{~km}$ (Dasgupta等, 2013). 如果综合考虑 $\mathrm{CO}_{2}$ 和水的效应，洋中脊之下地 幔橄榄岩的起始熔融深度将高达220 300km. 当然,



图 4 水和 $\mathrm{CO}_{2}$ 令地幔橄榄岩熔融温度降低

洋中脊和板内熔融的地幔位势温度(地幔沿绝热地温梯度线上涌但不发生焢融的地表温度)分别为 1350 和 $1500^{\circ} \mathrm{C}$; 含水橄榄岩固相线旁标注为 橄榄岩的总体水含量, 含碳橄榄岩固相线旁标注为熔体中的 $\mathrm{CO}_{2}$ 含量. 根据 Hirschmann(2006), Hirschmann 等(2009)和 Dasgupta 等(2013)修改 
洋中脊的岩浆作用 $(5 \sim 20 \mathrm{wt}$. \% 的部分熔融) 主要还是 发生在浅部, 深度超过 $65 \mathrm{~km}$ 层位地幔的部分熔融程 度较低, 一般不超过 $1 \mathrm{wt}$. \% . 在深部产生的是富含 $\mathrm{CO}_{2}$ 和水的玄武质熔体, 但熔体中的挥发分含量随着 深度的变浅和部分熔融程度的增大而降低.

与洋中脊之下的地幔橄榄岩相比, 对于板块内部 发生部分熔融形成洋岛玄武岩和大陆溢流玄武岩的情 况，一般认为该区域对应的地幔位势温度可能要高出 许多, 导致地幔在约 $120 \mathrm{~km}$ 深度就会穿越橄榄岩的干固 相线. 此外, 就洋岛玄武岩来说, 其源区地幔的水含量 一般为300 1000ppm, 高于MORB源区地幔的50 200 ppm(Dixon等, 2002). 图4显示, 含有600ppm水的橄榄 岩的固相线温度远低于热的板内地温梯度线, 板内部 分熔融很可能早在超过 $300 \mathrm{~km}$ 深度就已经发生了.

\section{3 水与地球深部的低度熔融}

目前对地球深部的认识基本上还处在一维模型 阶段, 即地球由不同的层圈构成, 层圈之间在矿物组 成或机械性质上存在明显差别, 但在横向上变化不 大. 与岩浆作用主要发生在板块边界可以类比的是, 地球深部的低程度熔融主要发生在层圈边界(Bercovici 和 Karato, 2003; Kawakatsu等, 2009; Schmandt等, 2014). 一般认为, 这是由于相邻层圈之间储水能力 (Water storage capacity)的差异造成的.

文献中(如Keppler, 2013) 常常将矿物与某种含水 液相处于热力学平衡时矿物中的水含量笼统地称为 水的溶解度(Water solubility), 但Hirschmann等(2005) 指出将其称为矿物的储水能力更为确切, 这一概念 逐渐得到推广. 下面以 $1 \mathrm{GPa}$ 下的镁橄榄石-水体系为 例, 解释狭义的水溶解度和矿物储水能力之间的区 别(图5). 在低温下与水的活度近似等于 1 的富水流体 共存时, 名义上无水矿物容纳的水含量为该矿物的 水溶解度, 即图 5 中的长划线. 但在高温下, 往矿物 中添加水至一定程度时会引发矿物熔融而非富水流 体的出溶, 此时所生成熔体的水含量低于与富水流体 共存的熔体(即水饱和熔体)水含量, 水的活度小于 1 . 图 5 中的短划线既是水不饱和条件下的橄榄石固相 线, 同时也对应Hirschmann等(2005)定义的矿物储水 能力. 储水能力的概念适用于各种温度和压强条件 (包括 $P>P_{\mathrm{c}}$ ), 指在不形成含水流体或熔体相的前提下

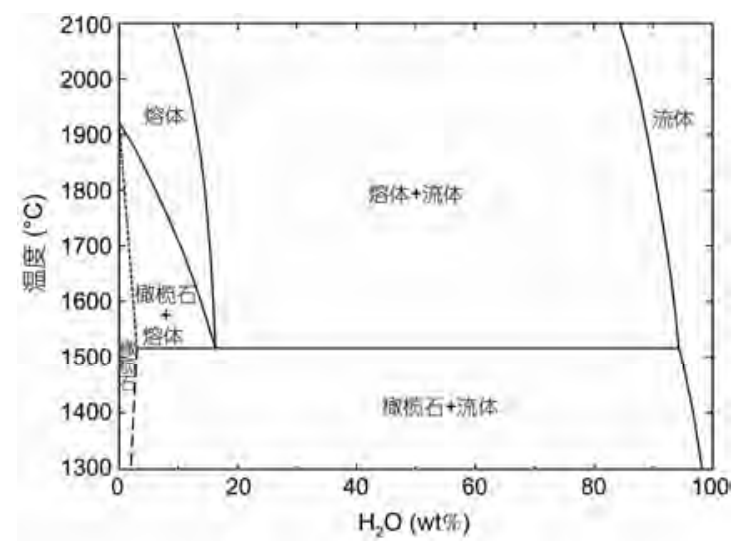

图 5 镁橄榄石 $-\mathrm{H}_{2} \mathrm{O}$ 体系在 $1 \mathrm{GPa}$ 下的相图

在低于水饱和固相线温度 $1520^{\circ} \mathrm{C}$ 时, 与橄榄石共存的是富水流体 (水的活度接近于 1), 长划线代表水在橄榄石中的溶解度. 在高于 $1520^{\circ} \mathrm{C}$ 时, 与橄榄石共存的是水不饱和的熔体(水的活度显著小于 1), 短划线代表橄榄石的储水能力. 储水能力的概念可以涵盖溶解 度. 为了清楚显示, 图中短划线和长划线指示的储水能力/溶解度有 所夸大. 根据 Hodges(1974)修改

矿物中能够储存的最大水含量.

如果在跨越层圈边界时矿物或岩石的储水能力 由高转低, 而其原本的实际水含量又大于转变后的 储水能力, 水必然从固相中逃逸出来, 从而在高温条 件下引发熔融．这样的情况至少可能发生在三处层 圈边界, 即岩石圈和软流圈边界、上地幔和过渡带边 界以及过渡带和下地幔边界。

\section{1 岩石圈和软流圈边界}

在岩石圈和软流圈边界(简称LAB)存在着一个 地震波低速带(简称LVZ), 在大洋板块下面的深度大 约为 80 220km, 大陆板块下面约为 $150 \sim 200 \mathrm{~km}$ (Anderson, 1989). 地震波速度和能量的衰减往往还 伴随着电导率异常(Evans等, 2005; Naif等, 2013). 有 研究者认为这种低速高导现象是由富水或富 $\mathrm{Fe}^{3+}$ 的 橄榄石和辉石造成的 (Karato, 2012; 杨晓志, 2013), 但单凭固相导电机制是否足以产生与地球物理观测 相符的结果仍存在争议(Yoshino等, 2006; Yoshino和 Katsura, 2013). 至少在部分地区, 这种异常可能与地 幔的低程度部分熔融有关(Anderson和Sammis，1970; Kawakatsu等, 2009). 根据对含 Al 斜方辉石的水溶解 度的实验研究, Mierdel等(2007)发现在低速带深度由 $60 \%$ 橄榄石 $+40 \%$ 铝饱和辉石所代表的地幔橄榄岩的 水溶解度出现一个低谷, 溶解度最低可以达到 700 
ppm左右(图6). 熔融可以在较低的水的活度(如0.1)下 发生, 因此100 200ppm的地幔水含量便足以引发熔 融. 因此, 他们认为低速带顶部名义上无水矿物地幔 水溶解度或储水能力的迅速下降导致了低速带的部 分熔融. Green等 $(2010,2014)$ 同样将低速带的部分熔 融归因为储水能力的变化, 但认为这是由浅部的含 水矿物(非闪石)在 $90 \mathrm{~km}$ 深度的分解造成的.

尽管在具体细节上仍存在一些分歧，但岩石圈 和软流圈边界可能存在少量 $(<1 \%)$ 富含 $\mathrm{H}_{2} \mathrm{O}$ 和 $\mathrm{CO}_{2}$ 的 玄武质熔体的看法获得了众多来自于高温高压实验 (Ni等, 2011; Dasgupta等, 2013; Sifré等, 2014)和地球 物理观测(Kawakatsu等, 2009; Naif等, 2013; Evans, 2014)方面的证据支持.

\section{2 上地幔和过渡带边界}

地球物理观测表明在 $410 \mathrm{~km}$ 深度, 即上地幔和 过渡带边界(简称UTB)也可能有熔体层存在(Revenaugh 和Sipkin, 1994; Toffelmier和Tyburczy, 2007; Tauzin 等, 2010). 林伍德石和瓦兹利石等过渡带矿物可以容 纳2 3wt.\%的水(Kohlstedt等, 1996), 远远超过橄榄石
和辉石等上地幔矿物在 $410 \mathrm{~km}$ 深度的储水能力 $(<0.2$ wt.\%; Ferot 和 Bolfan-Casanova，2012). Bercovici 和 Karato(2003)据此假设在410 km深度广泛存在熔体 层, 它在地球化学方面的作用相当于一个“水过滤 器”(Water filter), 即地幔物质从富水的过渡带上涌到 上地幔时发生普遍的脱水熔融, 导致包括水在内的 不相容组分被过滤而富集在熔体中, 余下的贫水地 幔物质继续上升并成为MORB的源区.

对 $410 \mathrm{~km}$ 深度条件下硅酸盐熔体密度的实验研 究表明, 熔体层在重力上应该是稳定的(Matsukage 等, 2005; Sakamaki等, 2006; Jing和Karato, 2012). 然 而, “水过滤器”假说面临的一个重大挑战在于, 过渡 带矿物的储水能力并不一定等同于过渡带的实际水 含量. 来自于过渡带的天然样品十分稀有, 而从上地 幔橄榄岩或幔源岩浆反推的地幔水含量一般仅为几 百ppm(Bell和Rossman, 1992). 不过, Pearson等(2014) 最近报道了首个天然林伍德石样品(巴西Juina金伯利 岩中金刚石所含的林伍德石包裹体)含有约 $1 \mathrm{wt} . \%$ 的 水. 如果可以证实这么高的水含量具有全球尺度上 的普遍性, 将为过渡带富水和UTB可能存在熔体层



图 6 地幔橄榄岩 $(60 \%$ 橄榄石 $+\mathbf{4 0 \%}$ 铝饱和辉石 $)$ 的水溶解度随深度的变化

(a) 为大陆板块, (b) 为大洋板块, 二者在地温梯度上存在较大差别(直至 $240 \mathrm{~km}$ 深度), 根据 Mierdel 等(2007)修改 
的认识提供有力支持.

\section{3 过渡带和下地幔边界}

关于下地幔两种主要组成矿物布里奇曼石(最近 有研究表明它在超过 $2000 \mathrm{~km}$ 深度可能分解为贫铁和 富铁的两相, 见Zhang等, 2014)和铁方镁石储水能力 的实验研究仍存在较大争议, 结果介于几 $\mathrm{ppm}$ 到 4000 ppm之间(Bolfan-Casanova等, 2002, 2003; Murakami 等, 2002; Litasov等, 2003). 无论如何, 只要不存在致 密含水镁硅酸盐矿物 (Dense Hydrous Magnesium Silicates, 简称DHMS), 下地幔的储水能力都远低于 过渡带, 这意味着在地幔对流过程中富水的过渡带 下沉到 $660 \mathrm{~km}$ 深度时很可能会引发熔融. 如果下地幔 的储水能力不足 $20 \mathrm{ppm}$, 即便是作为MORB源区的贫 水上地幔在下沉到 $660 \mathrm{~km}$ 深度时也会发生熔融. 当 然, 含有一定数量水的俯冲板片自身在到达过渡带 和下地幔边界(简称TLB)时也可能发生熔融(图7).

Schmandt等(2014)通过金刚石压腔实验证实, 含
水的林伍德石在分解为布里奇曼石和铁方镁石时会 发生脱水熔融. 他们同时还提供了地球物理方面的 证据，即在北美大陆下面地震波速在 $660 \mathrm{~km}$ 深度发生 显著降低. 浮力作用可能使过渡带和下地幔边界的 熔体向上渗透，使水返回过渡带. 因此, 410和660km 深度的脱水熔融可能使过渡带长期保持富水条件. 而下地幔是贫水区意味着它可能并非洋岛玄武岩 (OIB)的潜在源区. OIB 可能源自过渡带或上地幔中的 少量富水端元(图7), 如软流圈地幔中的地壳交代岩, 即贫橄榄石的辉石岩或角闪石岩(Zheng, 2012).

\section{4 水在地幔矿物和熔体之间的分配行为}

上文表明，无论是何种构造环境下的岩浆作用 还是地球内部层圈边界的低程度部分熔融(图7), 水 都对地幔的熔融发挥了显著的促进作用. 水可以促 进地幔岩石熔融的根本原因在于水是一种不相容组 分, 强烈倾向于富集在硅酸盐熔体相(矿物-熔体的水

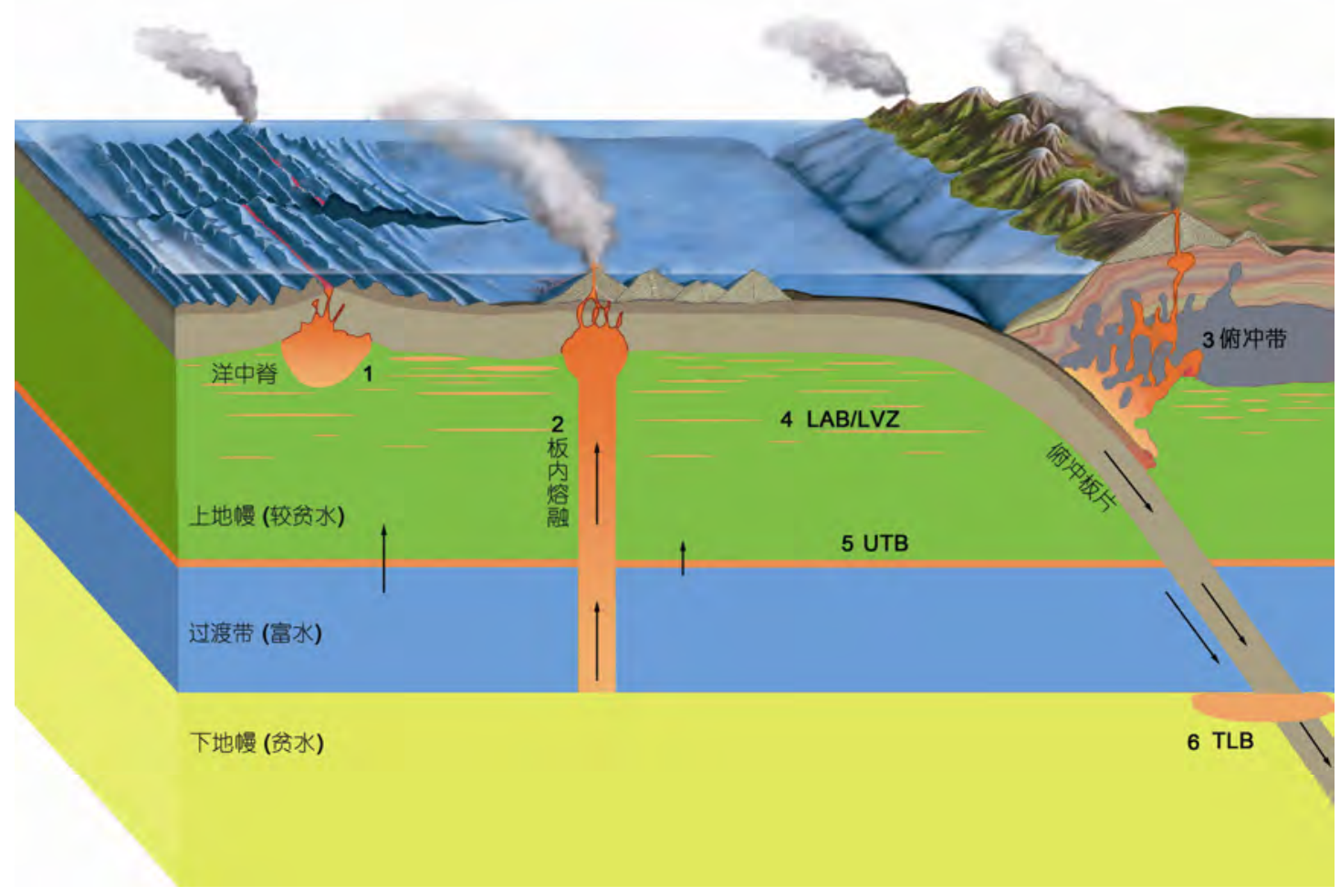

图 7 地球内部潜在的熔融区域

图中红色阴影区域代表不同的熔融区域: (1) 洋中脊; (2) 板内熔融; (3) 俯冲带地幔楔和俯冲洋壳; (4) 岩石圈-软流圈边界或软流圈低速带 (LAB/LVZ); (5) 上地幔和过渡带边界(UTB); (6) 过渡带和下地幔边界(TLB), 部分取材自大英百科全书图件 
分配系数远小于 1 ), 从而令其吉布斯自由能降低. 水 分配系数越小, 岩石的熔融温度就下降得越多, 熔融 比例也增大得越多; 一旦熔体脱离源区, 所产生的水 含量分异效应也就越显著.

水在岩石与熔体之间的分配系数是各单矿物与 熔体之间水分配系数的加权平均, 权重为矿物在固 相中的质量分数. 对于固定的矿物成分和熔体成分, 矿物-熔体的水分配系数 $D^{\mathrm{min} / \mathrm{melt}}$ 是温度、压强和水逸 度的函数, 其表达式为 (Keppler和Bolfan-Casanova, 2006; Keppler, 2013)

$$
D^{\min / \text { melt }}=\frac{C^{\min }}{C^{\text {melt }}}=A f_{\mathrm{H}_{2} \mathrm{O}}^{n} \exp \left(-\frac{\Delta H+P \Delta V}{R T}\right),
$$

式中, $C$ 代表矿物(min)或熔体(melt)的水含量(质量百分 数或 $\mathrm{ppm}$, 水逸度 $f_{\mathrm{H} 2 \mathrm{O}}$ 的指数 $n$ 取决于 $H$ 在矿物和熔体 中的赋存形式, $\Delta H$ 和 $\Delta V$ 分别代表矿物相与熔体相之间 水的偏摩尔焓和偏摩尔体积的差值, $R$ 为普适气体常数.

得益于低本底离子探针技术的快速发展, 针对 橄榄石、单斜辉石、斜方辉石和石榴石等主要地幔名 义上无水矿物与玄武质熔体之间水分配系数的实验 研究已经取得了长足进展(Koga等, 2003; Aubaud等, 2004, 2008; Hauri等, 2006; Tenner等, 2009, 2012; O’Leary等, 2010; Novella等, 2014). 在0.5 6GPa压强 下，辉石与熔体之间的水分配系数基本介于 0.01 0.03 , 它在等压条件下的波动主要反映了水分配系数 随辉石 $\mathrm{Al}$ 含量的增加而增大; 橄榄石或石榴石与熔 体之间的水分配系数要低一个数量级左右, 介于 0.001 0.004(图8). 在单矿物-熔体水分配系数基础上, Hirschmann等(2009)考虑矿物比例和矿物成分随压强 的变化, 确定了不同压强下地幔橄榄岩-熔体的水分 配系数. 全岩水分配系数在初始时随压强的升高而 增大, 在 $2.8 \mathrm{GPa}$ 达到 0.0085 的最大值, 这主要反映了 辉石 $\mathrm{Al}$ 含量的提升; 全岩-熔体水分配系数其后随压 强的升高而降低, 在上地幔底部降至0.003左右, 这 源自于辉石 $\mathrm{Al}$ 含量的降低与辉石向石榴石的转变(图 8). 当熔融程度增大时, 全岩-熔体水分配系数也会 发生变化. 由于富水的单斜辉石率先被消耗, 全岩熔体水分配系数一般随熔融的进行而降低.

\section{5 水对硅酸盐熔体物理性质的影响}

在熔融发生后，水富集在硅酸盐熔体相(含量一

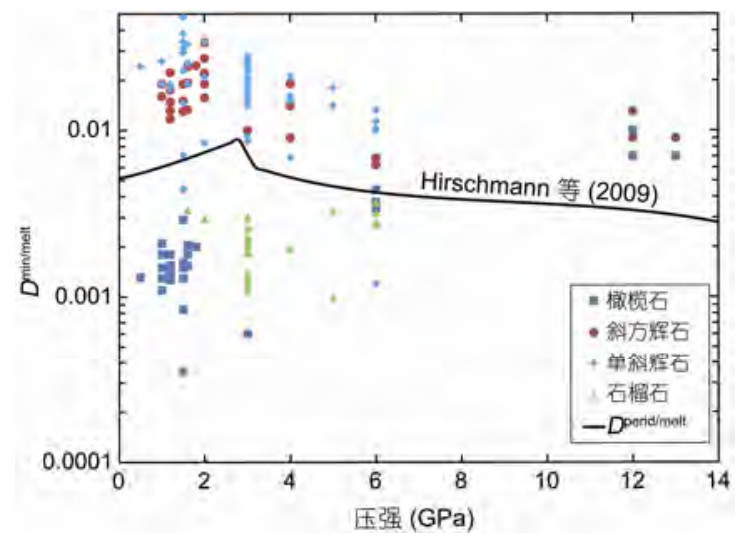

图 8 地幔名义上无水矿物与熔体之间的水分配系数 $\left(D^{\mathrm{min} / \mathrm{melt}}\right)$ 随压强的变化

图中数据点分别代表橄榄石、斜方辉石、单斜辉石以及石榴石与熔 体之间的水分配系数，出自 Koga 等(2003); Aubaud 等(2004，2008); Hauri 等(2006); Tenner 等(2009, 2012); O’Leary 等(2010); Novella 等 (2014); 实线代表地幔橄榄岩与熔体之间的总体水分配系数 (Hirschmann 等, 2009)

般在几千 ppm至wt.\%量级), 并使熔体的物理性质发 生显著变化(倪怀玮, 2013), 主要包括密度减小(Ochs 和Lange，1999)，黏度降低(Richet等，1996; Hui和 Zhang, 2007), 扩散系数增大(Watson, 1981; Zhang等, 2010), 电导率升高(Gaillard, 2004; Ni等, 2011; 郭璇 等, 2016)等.

在 $1000^{\circ} \mathrm{C}$ 和常压条件下, 水在硅酸盐熔体中的 偏摩尔体积为 $22.9 \mathrm{~cm}^{3} / \mathrm{mol}($ Ochs 和Lange, 1999), 小于 纯水流体的摩尔体积, 但它对应的偏摩尔密度仅为 $0.786 \mathrm{~g} / \mathrm{cm}^{3}$, 远小于干的熔体 $2 \sim 3 \mathrm{~g} / \mathrm{cm}^{3}$ 的密度. 在讨 论地球深部熔体的密度时, 水和 $\mathrm{FeO}$ 是最受重视的两 种组分(Matsukage等, 2005; Jing和Karato, 2012). 水 的存在增强了熔体的浮力效应, 使其更容易脱离源区 而独自演化; 而 $\mathrm{FeO}$ 使熔体密度增大, 有助于使熔体 长期停留在源区(如上地幔和过渡带边界的情况).

微量水的加入就可以使熔体的黏度发生数量级 的下降(Richet等, 1996; Audétat和Keppler, 2004). 原 始黏度越高, 加水的效果就越明显. 从微观角度看, 这与水的解聚作用有关, 即水分子与连接相邻硅氧四 面体的桥氧反应，生成两个羟基 $(\mathrm{OH}): \mathrm{H}_{2} \mathrm{O}+\mathrm{Si}-\mathrm{O}-\mathrm{Si}=$ $2 \mathrm{Si}-\mathrm{OH}$, 从而导致熔体的聚合度和黏度下降(Stolper, 1982; Malfait和Xue，2010). 水对熔体黏度和密度的 降低效应可以有效地促进熔体从其源区的分凝.

水也使各种离子在熔体中的扩散加快(Watson, 
1981; Zhang等, 2010). 就水自身来说, 除了水含量极 低的情况(如<100ppm), $\mathrm{H}_{2} \mathrm{O}$ 而非 $\mathrm{OH}$ 主导了水的扩散 (Zhang等, 1991; Ni等，2013). 就其他离子和原子来 说, 与 $\mathrm{Na}$ 和 $\mathrm{He}$ 等迁移快的元素相比, 水对 $\mathrm{Si}$ 和 $\mathrm{O}$ 等迁 移较慢的元素扩散系数的提升效果要显著得多. 扩散 系数的增大有利于促进晶体和气泡在岩浆中的生长.

水还可以显著提升熔体的电导率(Gaillard, 2004; Mookherjee等, 2008; 郭璇等, 2016). 将含水熔体电 导率实验测定与大地电磁测深相结合已经成为探测 地球内部熔融状态的一项重要手段(Ni等, 2011; Sifré 等, 2014), 甚至有望为火山喷发的预测提供一定依据.

\section{6 总结与展望}

水对地球上不同构造环境下和不同深度的各种 地幔熔融过程都发挥了显著的促进作用. 尽管在水 与地幔熔融的关系、水在地幔矿物和熔体之间的分配 以及水对于熔体物理性质的影响及其机制等研究方 面已经取得了很大进展, 但仍有很多重要的科学问 题亟待解决. 例如, 水饱和条件下橄榄岩和玄武岩固 相线的位置存在相当大的争议(图1和2), 对于不同温 度、压强、水含量和熔融程度下形成熔体的成分也知 之甚少. 玄武岩-水体系的第二临界端点的位置仍存 在较大的不确定性, 这直接关系到什么条件下才能 产生中间成分的超临界流体. 在水不饱和条件下, 少 量水对橄榄岩固相线的影响主要是通过水在矿物和 熔体之间的分配系数结合凝固点下降的方法来确定 的(Hirschmann, 2006; Hirschmann等, 2009), 其可靠 性还需要接受更多高温高压熔融实验结果(如Liu等, 2006)的检验. 地幔矿物与熔体之间的水分配系数随 矿物和熔体成分的变化规律仍有待更深入的考察. 对于地球深部极高温度和压强条件下含水熔体和超 临界流体的物理性质尚缺乏足够了解.

以上很多问题其实都受限于高温高压实验和分 析技术的发展. 含水镁铁质熔体和超临界流体无法 像含水长英质熔体那样在淬火过程中以玻璃的形式 保存下来. 即便含水熔体侥幸得以保存, 从低程度熔 融 $(<0.1 \mathrm{wt}$. \%) 实验产物中几乎不可能检测出极微量的 熔体(Hirschmann, 2006). 采用原位测试技术在成像 和物理性质方面较为可行, 但在成分分析方面仍力 有未逮. 实验产物中的矿物晶体尺寸有限, 用显微红
外光谱(FTIR)进行水含量分析时在空间分辨率方面 经常不能满足要求, 即使采用离子探针分析往往也 难以避免亚微米级杂质(熔体/流体/含水矿物包裹体) 的干扰. 将来一方面应继续加强实验和分析技术的 创新, 另一方面需要大力推进热力学、统计力学和分 子动力学计算和建模, 才有可能在地幔岩石体系的 相关系、水的分配系数与含水熔体和超临界流体的物 理性质等方面取得突破性进展.

致谢郑永飞主编约稿并提出了修改建议, 南京大学 杨晓志教授、北京大学刘琼副教授与一位匿名评审人提 出了修改建议, 在此一并致谢.

\section{参考文献}

郭璇, 陈琪, 倪怀玮. 2016. 含水硅酸盐熔体和富水流体电导率的 实验测定和应用. 中国科学: 地球科学, 46: 430-440

倪怀玮. 2013. 硅酸盐熔体的物理化学性质研究进展及其应用. 科 学通报, 58: 865-890

杨晓志. 2013. 上地幔顶部电导率异常的起因. 岩石矿物学杂志, 32: 663-679

Adam J, Locmelis M, Afonso J C, Rushmer T, Fiorentini M L. 2014. The capacity of hydrous fluids to transport and fractionate incompatible elements and metals within the Earth's mantle. Geochem Geophys Geosyst, 15: 2241-2253

Anderson D L, Sammis C. 1970. Partial melting in the upper mantle. Phys Earth Planet Inter, 3: 41-50

Anderson D L. 1989. Theory of the Earth. Blackwell Scientific Publications. 366

Aubaud C, Hauri E H, Hirschmann M M. 2004. Hydrogen partition coefficients between nominally anhydrous minerals and basaltic melts. Geophys Res Lett, 31: L20611, doi: 10.1029/2004GL021341

Aubaud C, Hirschmann M M, Withers A C, Hervig R L. 2008. Hydrogen partitioning between melt, clinopyroxene, and garnet at $3 \mathrm{GPa}$ in a hydrous MORB with $6 \mathrm{wt} \% \mathrm{H}_{2} \mathrm{O}$. Contrib Mineral Petrol, 156: 607-625

Audétat A, Keppler H. 2004. Viscosity of fluids in subduction zones. Science, 303: 513-516

Bell D R, Rossman G R. 1992. Water in Earth's mantle: The role of nominally anhydrous minerals. Science, 255: 1391-1397

Beran A, Libowitzky E. 2006. Water in natural mantle minerals II: Olivine, garnet and accessory minerals. Rev Mineral Geochem, 62: 169-191

Bercovici D, Karato S I. 2003. Whole-mantle convection and the transition-zone water filter. Nature, 425: 39-44

Bolfan-Casanova N, Keppler H, Rubie D C. 2003. Water partitioning at $660 \mathrm{~km}$ depth and evidence for very low water solubility in 
magnesium silicate perovskite. Geophys Res Lett, 30: 1905, doi: 10.1029/2003GL017182

Bolfan-Casanova N, Mackwell S, Keppler H, McCammon C, Rubie D C. 2002. Pressure dependence of $\mathrm{H}$ solubility in magnesiowüstite up to $25 \mathrm{GPa}$ : Implications for the storage of water in the Earth's lower mantle. Geophys Res Lett, 29: 1449, doi: 10.1029/2001GL014457

Dasgupta R. 2013. Ingassing, storage, and outgassing of terrestrial carbon through geologic time. Rev Geophys Res, 75: 183-229

Dasgupta R, Mallik A, Tsuno K, Withers A C, Hirth G, Hirschmann M M. 2013. Carbon-dioxide-rich silicate melt in the Earth's upper mantle. Nature, 493: 211-215

Defant M J, Drummond M S. 1990. Derivation of some modern arc magmas by melting of young subducted lithosphere. Nature, 347 : 662-665

Dixon J E, Leist L, Langmuir C, Schilling J G. 2002. Recycled dehydrated lithosphere observed in plume-influenced mid-oceanridge basalt. Nature, 420: 385-389

Evans R L. 2014. Geophysics: Making the Earth move. Nature, 509: $40-41$

Evans R L, Hirth G, Baba K, Forsyth D, Chave A, Mackie R. 2005. Geophysical evidence from the MELT area for compositional controls on oceanic plates. Nature, 437: 249-252

Ferot A, Bolfan-Casanova N. 2012. Water storage capacity in olivine and pyroxene to $14 \mathrm{GPa}$ : Implications for the water content of the Earth's upper mantle and nature of seismic discontinuities. Earth Planet Sci Lett, 349: 218-230

Gaillard F. 2004. Laboratory measurements of electrical conductivity of hydrous and dry silicic melts under pressure. Earth Planet Sci Lett, 218: 215-228

Green D H, Hibberson W O, Kovács I, Rosenthal A. 2010. Water and its influence on the lithosphere-asthenosphere boundary. Nature, 467: 448-451

Green D H, Hibberson W O, Rosenthal A, Kovács I, Yaxley G M, Falloon T J, Brink F. 2014. Experimental study of the influence of water on melting and phase assemblages in the upper mantle. $\mathrm{J}$ Petrol, 55: 2067-2096

Grove T L, Chatterjee N, Parman S W, Médard E. 2006. The influence of $\mathrm{H}_{2} \mathrm{O}$ on mantle wedge melting. Earth Planet Sci Lett, 249: 74-89

Hack A C, Thompson A B, Aerts M. 2007. Phase relations involving hydrous silicate melts, aqueous fluids, and minerals. Rev Mineral Geochem, 65: 129-185

Hauri E H, Gaetani G A, Green T H. 2006. Partitioning of water during melting of the Earth's upper mantle at $\mathrm{H}_{2} \mathrm{O}$-undersaturated conditions. Earth Planet Sci Lett, 248: 715-734

Hermann J, Spandler C, Hack A, Korsakov A V. 2006. Aqueous fluids and hydrous melts in high-pressure and ultra-high pressure rocks: Implications for element transfer in subduction zones. Lithos, 92: 399-417
Hirschmann M M. 2000. Mantle solidus: experimental constraints and the effects of peridotite composition. Geochem Geophys Geosyst, 1, doi: 10.1029/2000GC000070

Hirschmann M M. 2006. Water, melting, and the deep Earth $\mathrm{H}_{2} \mathrm{O}$ cycle. Annu Rev Earth Planet Sci, 34: 629-653

Hirschmann M M, Aubaud C, Withers A C. 2005. Storage capacity of $\mathrm{H}_{2} \mathrm{O}$ in nominally anhydrous minerals in the upper mantle. Earth Planet Sci Lett, 236: 167-181

Hirschmann M M, Tenner T, Aubaud C, Withers A C. 2009. Dehydration melting of nominally anhydrous mantle: The primacy of partitioning. Phys Earth Planet Inter, 176: 54-68

Hodges F. 1974. The solubility of $\mathrm{H}_{2} \mathrm{O}$ in silicate melts. Year Book Carnegie Inst Washington, 73: 251-255

Hui H, Zhang Y. 2007. Toward a general viscosity equation for natural anhydrous and hydrous silicate melts. Geochim Cosmochim Acta, 71: 403-416

Jing Z, Karato S I. 2012. Effect of $\mathrm{H}_{2} \mathrm{O}$ on the density of silicate melts at high pressures: Static experiments and the application of a modified hard-sphere model of equation of state. Geochim Cosmochim Acta, 85: 357-372

Johnson E A. 2006. Water in nominally anhydrous crustal minerals: speciation, concentration, and geologic significance. Rev Mineral Geochem, 62: 117-154

Karato S I. 2012. On the origin of the asthenosphere. Earth Planet Sci Lett, 321-322: 95-103

Katz R F, Spiegelman M, Langmuir C H. 2003. A new parameterization of hydrous mantle melting. Geochem Geophys Geosyst, 4: 1073, doi: 10.1029/2002GC000433

Kawakatsu H, Kumar P, Takei Y, Shinohara M, Kanazawa T, Araki E, Suyehiro K. 2009. Seismic evidence for sharp lithosphereasthenosphere boundaries of oceanic plates. Science, 324: 499502

Keppler H. 2013. Volatiles under high pressure. In: Shun-Ichiro Karato, ed. Physics and Chemistry of the Deep Earth. Wiley. 3-37

Keppler H, Bolfan-Casanova N. 2006. Thermodynamics of water solubility and partitioning. Rev Mineral Geochem, 62: 193-230

Kessel R, Ulmer P, Pettke T, Schmidt M W, Thompson A B. 2005a. The water-basalt system at 4 to $6 \mathrm{GPa}$ : Phase relations and second critical endpoint in a $\mathrm{K}$-free eclogite at 700 to $1400^{\circ} \mathrm{C}$. Earth Planet Sci Lett, 237: 873-892

Kessel R, Schmidt M W, Ulmer P, Pettke T. 2005b. Trace element signature of subduction-zone fluids, melts and supercritical liquids at $120-180 \mathrm{~km}$ depth. Nature, $437: 724-727$

Kimura J I, Nakajima J. 2014. Behaviour of subducted water and its role in magma genesis in the NE Japan arc: A combined geophysical and geochemical approach. Geochim Cosmochim Acta, 143: 165-188

Koga K, Hauri E H, Hirschmann M M, Bell D. 2003. Hydrogen concentration analyses using SIMS and FTIR: Comparison and calibration for nominally anhydrous minerals. Geochem Geophys 
Geosyst, 4: 1019, doi: 10.1029/2002GC000378

Kohlstedt D, Keppler H, Rubie D. 1996. Solubility of water in the $\alpha, \beta$ and $\gamma$ phases of $(\mathrm{Mg}, \mathrm{Fe})_{2} \mathrm{SiO}_{4}$. Contrib Mineral Petrol, 123: $345-357$

Lambert I B, Wyllie P J. 1972. Melting of a gabbro (qtz eclogite) with excess $\mathrm{H}_{2} \mathrm{O}$ to 35 kbars with geological applications. J Geol, 80: 693-708.

Litasov K, Ohtani E, Langenhorst F, Yurimoto H, Kubo T, Kondo T. 2003. Water solubility in Mg-perovskites and water storage capacity in the lower mantle. Earth Planet Sci Lett, 211: 189-203

Liu X, O’Neill H S C, Berry A J. 2006. The effects of small amounts of $\mathrm{H}_{2} \mathrm{O}, \mathrm{CO}_{2}$ and $\mathrm{Na}_{2} \mathrm{O}$ on the partial melting of spinel lherzolite in the system $\mathrm{CaO}-\mathrm{MgO}-\mathrm{Al}_{2} \mathrm{O}_{3}-\mathrm{SiO}_{2} \pm \mathrm{H}_{2} \mathrm{O} \pm \mathrm{CO}_{2} \pm \mathrm{Na}_{2} \mathrm{O}$ at $1.1 \mathrm{GPa}$. J Petrol, 47: 409-434

Malfait W J, Xue X. 2010. The nature of hydroxyl groups in aluminosilicate glasses: Quantifying Si-OH and Al-OH abundances along the $\mathrm{SiO}_{2}-\mathrm{NaAlSiO}_{4}$ join by ${ }^{1} \mathrm{H},{ }^{27} \mathrm{Al}-{ }^{1} \mathrm{H}$ and ${ }^{29} \mathrm{Si}-{ }^{1} \mathrm{H} \mathrm{NMR}$ spectroscopy. Geochim Cosmochim Acta, 74: 719-737

Manning C E. 2004. The chemistry of subduction-zone fluids. Earth Planet Sci Lett, 223: 1-16

Matsukage K N, Jing Z, Karato S I. 2005. Density of hydrous silicate melt at the conditions of Earth's deep upper mantle. Nature, 438: 488-491

Mibe K, Kawamoto T, Matsukage K N, Fei Y, Ono S. 2011. Slab melting versus slab dehydration in subduction-zone magmatism. Proc Natl Acad Sci USA, 108: 8177-8182

Michael P. 1995. Regionally distinctive sources of depleted MORB: evidence from trace elements and $\mathrm{H}_{2} \mathrm{O}$. Earth Planet Sci Lett, 131: 301-320

Mierdel K, Keppler H, Smyth J R, Langenhorst F. 2007. Water solubility in aluminous orthopyroxene and the origin of Earth's asthenosphere. Science, 315: 364-368

Mookherjee M, Stixrude L, Karki B B. 2008. Hydrous silicate melt at high pressure. Nature, 452: 983-986

Murakami M, Hirose K, Yurimoto H, Nakashima S, Takafuji N. 2002. Water in Earth's lower mantle. Science, 295: 1885-1887

Naif S, Key K, Constable S, Evans R L. 2013. Melt-rich channel observed at the lithosphere-asthenosphere boundary. Nature, 495 : 356-359

Ni H, Keppler H, Behrens H. 2011. Electrical conductivity of hydrous basaltic melts: Implications for partial melting in the upper mantle. Contrib Mineral Petrol, 162: 637-650

$\mathrm{Ni} \mathrm{H,} \mathrm{Xu} \mathrm{Z,} \mathrm{Zhang} \mathrm{Y.} \mathrm{2013.} \mathrm{Hydroxyl} \mathrm{and} \mathrm{molecular} \mathrm{H}_{2} \mathrm{O}$ diffusivity in a haploandesitic melt. Geochim Cosmochim Acta, 103: 36-48

Novella D, Frost D J, Hauri E H, Bureau H, Raepsaet C, Roberge M. 2014. The distribution of $\mathrm{H}_{2} \mathrm{O}$ between silicate melt and nominally anhydrous peridotite and the onset of hydrous melting in the deep upper mantle. Earth Planet Sci Lett, 400: 1-13

O'Leary J A, Gaetani G A, Hauri E H. 2010. The effect of tetrahedral $\mathrm{Al}^{3+}$ on the partitioning of water between clinopyroxene and silicate melt. Earth Planet Sci Lett, 297: 111-120

Ochs F A, Lange R A. 1999. The density of hydrous magmatic liquids. Science, 283: 1314-1317

Pearson D, Brenker F, Nestola F, McNeill J, Hutchison M T, Matveev K, Mather K, Silvermit G, Schmitz S, Vekemans B, Vincze L. 2014. Hydrous mantle transition zone indicated by ringwoodite included within diamond. Nature, 507: 221-224

Poli S, Schmidt M W. 2002. Petrology of subducted slabs. Annu Rev Earth Planet Sci, 30: 207-235

Prouteau G, Scaillet B, Pichavant M, Maury R. 2001. Evidence for mantle metasomatism by hydrous silicic melts derived from subducted oceanic crust. Nature, 410: 197-200

Revenaugh J, Sipkin S. 1994. Seismic evidence for silicate melt atop the 410-km mantle discontinuity. Nature, 369: 474-476

Richet P, Lejeune A M, Holtz F, Roux J. 1996. Water and the viscosity of andesite melts. Chem Geol, 128: 185-197

Rumble D, Liou J G, Jahn B M. 2003. Continental crust subduction and ultrahigh pressure metamorphism. Treatise Geochem, 3: 293-319

Sakamaki T, Suzuki A, Ohtani E. 2006. Stability of hydrous melt at the base of the Earth's upper mantle. Nature, 439: 192-194

Salters V J M, Hart S R. 1989. The hafnium paradox and the role of garnet in the source of mid-ocean-ridge basalts. Nature, 342: 420-422

Salters V J M, Stracke A. 2004. Composition of the depleted mantle. Geochem Geophys Geosyst, 5: Q05004, doi: 10.1029/2003GC000597

Schmandt B, Jacobsen S D, Becker T W, Liu Z, Dueker K G. 2014. Dehydration melting at the top of the lower mantle. Science, 344 : 1265-1268

Shen A H, Keppler H. 1997. Direct observation of complete miscibility in the albite- $\mathrm{H}_{2} \mathrm{O}$ system. Nature, 385: 710-712

Sifré D, Gardés E, Massuyeau M, Hashim L, Hier-Majumder S, Gaillard F. 2014. Electrical conductivity during incipient melting in the oceanic low-velocity zone. Nature, 509: 81-85

Skogby H. 2006. Water in natural mantle minerals I: Pyroxenes. Rev Mineral Geochem, 62: 155-167

Smyth J R. 2006. Hydrogen in high pressure silicate and oxide mineral structures. Rev Mineral Geochem, 62: 85-115

Stern R J. 2002. Subduction zones. Rev Geophys, 40: 1012, doi: 10.1029/2001RG000108

Stolper E M. 1982. The speciation of water in silicate melts. Geochim Cosmochim Acta, 46: 2609-2620

Tauzin B, Debayle E, Wittlinger G. 2010. Seismic evidence for a global low-velocity layer within the Earth's upper mantle. Nature Geosci, 3: 718-721

Tenner T J, Hirschmann M M, Withers A C, Ardia P. 2012. $\mathrm{H}_{2} \mathrm{O}$ storage capacity of olivine and low-Ca pyroxene from 10 to 13 GPa: Consequences for dehydration melting above the transition zone. Contrib Mineral Petrol, 163: 297-316

Tenner T J, Hirschmann M M, Withers A C, Hervig R L. 2009. Hydrogen partitioning between nominally anhydrous upper mantle minerals and melt between 3 and $5 \mathrm{GPa}$ and applications to 
hydrous peridotite partial melting. Chem Geol, 262: 42-56

Till C B, Grove T L, Withers A C. 2012. The beginnings of hydrous mantle wedge melting. Contrib Mineral Petrol, 163: 669-688

Toffelmier D A, Tyburczy J A. 2007. Electromagnetic detection of a 410-km-deep melt layer in the southwestern United States. Nature, 447: 991-994

Watson E B. 1981. Diffusion in magmas at depth in the earth: the effects of pressure and dissolved $\mathrm{H}_{2} \mathrm{O}$. Earth Planet Sci Lett, 52: 291-301

Workman R K, Hart S R. 2005. Major and trace element composition of the depleted MORB mantle (DMM). Earth Planet Sci Lett, 231: $53-72$

Yoshino T, Katsura T. 2013. Electrical conductivity of mantle minerals: Role of water in conductivity anomalies. Annu Rev Earth Planet Sci, 41: 605-628

Yoshino T, Matsuzaki T, Yamashita S, Katsura T. 2006. Hydrous olivine unable to account for conductivity anomaly at the top of the asthenosphere. Nature, 443: 973-976

Zhang L, Meng Y, Yang W, Wang L, Mao W L, Zeng Q S, Jeong J S,
Wagner A J, Mkhoyam A, Liu W, Xu R, Mao H K. 2014. Disproportionation of $(\mathrm{Mg}, \mathrm{Fe}) \mathrm{SiO}_{3}$ perovskite in Earth's deep lower mantle. Science, 344: 877-882

Zhang Y X, Ni H, Chen Y. 2010. Diffusion data in silicate melts. Rev Mineral Geochem, 72: 311-408

Zhang Y X, Stolper E M, Wasserburg G. 1991. Diffusion of water in rhyolitic glasses. Geochim Cosmochim Acta, 55: 441-456

Zheng Y F, Fu B, Gong B, Li L. 2003. Stable isotope geochemistry of ultrahigh pressure metamorphic rocks from the Dabie-Sulu orogen in China: Implications for geodynamics and fluid regime. Earth Sci Rev, 62: 105-161

Zheng Y F, Xia Q X, Chen R X, Gao X Y. 2011. Partial melting, fluid supercriticality and element mobility in ultrahigh-pressure metamorphic rocks during continental collision. Earth Sci Rev, 107: 342-374

Zheng Y F. 2012. Metamorphic chemical geodynamics in continental subduction zones. Chem Geol, 328: 5-48

Zheng Y F, Hermann J. 2014. Geochemistry of continental subduction-zone fluids. Earth Planet Space, 66: 93 